\title{
Long-term safety of posterior chamber implantable phakic contact lens for the correction of myopia
}

This article was published in the following Dove Press journal:

Clinical Ophthalmology

\section{Gitansha Sachdev Dandapani Ramamurthy}

The Eye Foundation, Coimbatore, India
Correspondence: Gitansha Sachdev The Eye Foundation, 582-A, DB Road, RS Puram, Coimbatore 641002, India Tel +9l 9840826106 Email sachdevgitansha@gmail.com
Purpose: To review the safety of the implantable phakic contact lens (IPCL V1, Caregroup Sight Solutions, India) for correction of myopia and myopic astigmatism.

Methods: Retrospective interventional case series including 134 eyes which underwent IPCL implantation for correction of myopia and myopic astigmatism at a tertiary eye care hospital in South India. Intraoperative and postoperative complications were analyzed. The following parameters were analyzed at preoperative and 1,6 and 12 month postoperative visits: visual acuity (in $\log M A R$ ) and manifest refraction, slit lamp bio microscopy, dilated fundus examination, IOP and endothelial cell density measurement.

Results: No intraoperative complications were noted. Cataract was the most common postoperative complication, wherein three eyes $(2.2 \%)$ underwent observation for visually insignificant anterior subcapsular cataract and one eye $(0.7 \%)$ required surgical intervention. Four eyes developed transient increased IOP due to steroid response ( 3 eyes) and pupillary block glaucoma ( 1 eye). The endothelial cell loss noted over a 1 -year follow-up was $2.01 \% \pm 4.12 \%(P=0.71)$. One eye developed hypopyon on the third postoperative day, which resolved subsequently with an unaided vision of 20/20. No vision threatening complications were noted. The mean follow-up in the cohort was $25.66 \pm 16.45$ months.

Conclusion: The IPCL is a safe and effective treatment modality for correction of myopia and myopic astigmatism.

Keywords: phakic intraocular lens, implantable collamer lens, ICL, IPCL, implantable phakic contact lens, myopia, myopic astigmatism, refractive correction

\section{Introduction}

The phakic posterior chamber intraocular lens demonstrates various advantages over keratorefractive procedures for refractive correction. The advantages are more pronounced for higher levels of ametropia, with lower induction of postoperative aberrations, retinal image magnification and better contrast sensitivity. ${ }^{1-3}$ Preservation of accommodation is a distinct advantage over other modalities of treatment such as clear lens extraction. ${ }^{4}$

However, as an intraocular procedure, it is associated with a greater risk of complications including potential damage to anterior segment structures, retinal detachment and infections such as endophthalmitis.

The safety and efficacy of the Visian implantable collamer lens (ICL - Staar Surgical AG, Nidau, Switzerland) has been demonstrated for moderate and high ametropia over long-term follow-up. ${ }^{5-8}$ A limitation of the ICL, however, would be the economic burden of treatment, especially in developing nations. The Implantable Phakic Contact 
Lens (IPCL V1, Caregroup Sight Solutions, India) has been developed as an alternative option for refractive correction, at a distinct economic advantage. Additionally, the maximum afforded myopic correction which is readily available with ICL is limited to $-18.0 \mathrm{D}$. Higher ametropia although rare requires customized implants with associated increased costs. On the contrary, readily available IPCL can provide correction for a wide range of ametropia up to $-30.0 \mathrm{D}$.

We demonstrate the safety profile of the IPCL over a minimum follow-up period of 1 year. To the best of our knowledge, no similar studies have been published in literature thus far.

\section{Patients and methods Study population}

This retrospective study was conducted at a tertiary eye care hospital in South India. The protocol was registered and approved by the Ethics Committee of our Institute (Ethics Committee of The Eye Foundation, Coimbatore Registration Number: ECR/934/Inst/TN/2017) and adhered to the tenets of the Declaration of Helsinki. We included patients who underwent IPCL implantation for myopic correction between August 2014 and July 2017, by a single surgeon with a minimum follow-up period of 1 year.

Inclusion criteria were as follows: stable refraction (change in mean refractive spherical equivalent of not more than $0.25 \mathrm{D}$ ) for a minimum period of 1 year, age greater than 21 years, endothelial cell count $\geq 2,500$ cells $/ \mathrm{mm}^{2}$ and anterior chamber depth $\geq 2.8 \mathrm{~mm}$. Eyes with prior ocular surgery, comorbidities including cataract, glaucoma, uveitis and corneal ectasia were excluded from the study.

The following parameters were compared preoperatively and at 1, 6 and 12 months' postoperative visit: logarithm of the minimal angle of resolution (logMAR) of uncorrected distance visual acuity (UDVA), logMAR of corrected distance visual acuity (CDVA), manifest refractive error (spherical equivalent), IOP using Goldmann applanation tonometry and endothelial cell density (ECD) using specular microscopy (Topcon SP-1A).

All eyes underwent a slit-lamp biomicroscopic and dilated fundus evaluation. Preoperatively, the horizontal white-towhite was measured using the laser interferometry biometer (Lenstar, Haag Streit, USA) and handheld digital calipers. Anterior chamber depth (distance from corneal endothelium to anterior lens capsule) and keratometric values were obtained using Scheimpflung corneal tomography (Pentacam HR; Oculus Optikgerate GmbH, Wetzlar, Germany).
Eyes wherein the manifest cylinder was $-1.0 \mathrm{D}$ or less underwent non-toric implant (39 eyes), while the remaining underwent toric IPCL implantation (95 eyes). The phakic intraocular lens power was calculated using a modified vertex formula as per the manufacturer's recommendation, with a target refraction of emmetropia. The implant size was selected based on the anterior chamber depth and the horizontal white-to-white.

Preoperative peripheral iridotomies (at 10 or 2 o' clock) were done using the neodymium-yttrium garnet (Nd:YAG) laser preoperatively.

IPCL is a hydrophilic hybrid acrylic implant, with six haptic pads for better stability in the ciliary sulcus. Customization of the implant allows treatment for a wide range of ametropia (+15 D to $-30 \mathrm{D}$ in $0.5 \mathrm{D}$ increments) and astigmatism (up to $-10 \mathrm{D}$ in $0.5 \mathrm{D}$ increments). The lens design includes eight holes: two in the haptics, four along the optic haptic transition zone and two along the optic periphery to determine correct orientation. The IPCL (version 1) was commercially introduced in 2013 and at present is distributed worldwide in 20 countries.

\section{Surgical procedure}

A written informed consent was obtained from all patients. All surgeries were performed by a single experienced surgeon (DR). Topical anesthetic (proparacaine hydrochloride ophthalmic solution $0.5 \%$ ) and mydriatic agents (tropicamide $0.8 \%$ and phenylephrine hydrochloride $5 \%$ ) were instilled prior to the procedure. The phakic posterior chamber intraocular lens was implanted into the anterior chamber through a $3 \mathrm{~mm}$ clear corneal incision following ophthalmic viscoelastic device (HEALON OVD, sodium hyaluronate, Johnson \& Johnson Vision, Santa Ana, CA, USA) injection. The footplates were subsequently tucked behind the iris, followed by a thorough OVD wash. Digital image guided system (Verion image guided system; Alcon, Fort Worth, TX, USA) allowed measurement of intraoperative cyclotorsion and correct placement of the toric implants. Customization of the toric IPCL allows placement of the implant at the $0^{\circ}-180^{\circ}$ horizontal axis for all cases.

Postoperative treatment regimen included steroids (loteprednol eye drops $0.5 \%$ ) in tapering doses and antibiotic drops (moxifloxacin ophthalmic solution $0.5 \%$ ) and antiglaucoma medication (timolol maleate eye drops $0.5 \%$ ) for 2 weeks.

\section{Statistical analyses}

SPSS 12.0 for IBM (SPSS Inc., Chicago, IL, USA) was used for statistical analyses. Student's $t$-test was used for normally distributed variables, the Kruskal-Wallis test for nonparametric 
variables, and the chi-squared or Fisher's exact tests as indicated for the analysis of categorical variables. Snellen best-corrected visual acuity measurements were converted to logarithm of the minimum angle of resolution ( $\log M A R)$ equivalents for the purpose of data analysis. Data is presented as mean $\pm \mathrm{SD}$, or as $\mathrm{n}(\%)$ for categorical variables. A $P$-value of $<0.05$ was considered statistically significant.

\section{Results}

\section{Patient demographics}

Preoperative demographics of the study population are summarized in Table 1.

\section{Visual outcomes}

Figure 1 demonstrates the visual outcomes over a 1-year follow-up period.

Median logMAR of CDVA at 1-year postoperative visit was 0 (IQR 0,0.10). At 1-year postoperative visit, $65 \%$ of the eyes showed no change in CDVA, whereas $30 \%$ of the eyes gained 1 or more lines. Median $\log$ MAR of UDVA was 0.10 (IQR $0,0.18$ ) with an uncorrected visual acuity of 20/32 or better in $85 \%$ of the eyes. $88 \%$ and $95.8 \%$ of eyes were within $0.5 \mathrm{D}$ and $1.0 \mathrm{D}$ of the attempted correction following IPCL implantation.

\section{Complications or adverse effects}

No intraoperative complications were noted in any of the eyes.

\section{Immediate postoperative complications}

A shallow anterior chamber was noted in one eye $(0.74 \%)$ at first postoperative day secondary to a wound leak (superior

Table I Preoperative demographics of patients undergoing implantable posterior contact lens implantation

\begin{tabular}{|l|l|l|}
\hline Characteristics & Mean \pm SD & Range \\
\hline Age (years) & $25.28 \pm 4.24$ & 2 I to $4 \mathrm{I}$ \\
\hline Sphere $(\mathrm{D})$ & $-9.45 \pm 4.3 \mathrm{I}$ & $-\mathrm{I} .0$ to -22.0 \\
\hline Cylinder $(\mathrm{D})$ & $-1.72 \pm \mathrm{I} .26$ & 0 to -6.0 \\
\hline Manifest spherical equivalent $(\mathrm{D})$ & $-10.3 \mathrm{I} \pm 4.3 \mathrm{I}$ & $-\mathrm{I} .25$ to -25.0 \\
\hline Anterior chamber depth $(\mathrm{mm})$ & $3.2 \mathrm{I} \pm 0.28$ & $2.8 \mathrm{I}$ to 3.96 \\
\hline Flat keratometry $(\mathrm{D})$ & $43.60 \pm \mathrm{I} .44$ & 40.5 to 46.75 \\
\hline Steep keratometry $(\mathrm{D})$ & $45.15 \pm \mathrm{I} .6 \mathrm{I}$ & $4 \mathrm{I} .0$ to 49.0 \\
\hline Central corneal thickness $(\mu \mathrm{m})$ & $509.8 \mathrm{I} \pm 4 \mathrm{I} .32$ & 423 to 606 \\
\hline White to white $(\mathrm{mm})$ & $\mathrm{II} .59 \pm \mathrm{I} .06$ & $\mathrm{II} .03$ to $\mathrm{I} 2.55$ \\
\hline Endothelial cell density $\left(\mathrm{mm}^{2}\right)$ & $2,754.58 \pm 235.94$ & $2,5 \mathrm{I} 0$ to 3,353 \\
\hline Axial length $(\mathrm{mm})$ & $26.56 \pm \mathrm{I} .78$ & 23.23 to 32.8 \\
\hline
\end{tabular}

side port) with a positive Seidel's test. Anterior segment optical coherence tomography (ASOCT) revealed an adequate lens vault $(465 \mu \mathrm{m})$. The patient underwent anterior chamber reformation with a suture placement (10-0 nylon monofilament) to maintain the integrity of the side port wound. The patient achieved a UDVA of 20/20 by the fifth day of postoperative visit, which remained stable up to the last follow-up at 72 weeks.

One eye $(0.74 \%)$ demonstrated a dense anterior segment inflammation (trace hypopyon) on the fourth postoperative day, presenting as blurred vision and redness in the affected eye. The patient responded to intracameral antibiotic wash (moxifloxacin $0.5 \%$ ) and topical steroids (prednisolone acetate $0.1 \%$ suspension). He achieved a UDVA of 20/20 at 2 weeks' postoperative visit.

\section{Cataract formation}

Four eyes (2.9\%) developed an anterior subcapsular cataract (ASC). A higher mean age group (34.52 \pm 6.16 years) and preoperative manifest refraction $(-17.5 \pm 4.81$, range -11 to $-23.0 \mathrm{D})$ were noted in this subgroup. In all eyes, the initial cataract developed within 1 year of IPCL implantation. The lens vault was adequate in all eyes with a mean vault of 369.75 $475.65 \mu \mathrm{m}$ (range 259-429). One eye (0.74\%) required surgical intervention due to visually significant cataract (loss of 4 lines of CDVA) at 62 weeks' postoperative visit. The CDVA following cataract extraction with intraocular lens implantation was 20/25.

\section{Raised IOP}

Three eyes $(2.23 \%)$ developed moderately high IOP rise (mean $30.34 \pm 0.58 \mathrm{mmHg}$ ) due to steroid use. They were managed conservatively with steroid discontinuation and antiglaucoma medications, following which IOP normalized. One eye $(0.74 \%)$ required a repeat $\mathrm{Nd}$ :YAG peripheral iridotomy to enlarge the preexisting inadequate opening and relieve the pupillary block. No cases of pupillary block secondary to excessive lens vault were noted.

\section{ECD}

The central endothelial cell loss noted over a 1-year follow-up period was $2.01 \% \pm 4.12 \%(P=0.071)$.

\section{Long-term stability and rotation}

Six eyes $(4.47 \%)$ showed significant IPCL rotation $\left(5^{\circ}\right.$ or more) with a mean rotation of $10.67^{\circ} \pm 4.97^{\circ}\left(\right.$ range $\left.5^{\circ}-17^{\circ}\right)$. The anterior chamber depth (mean $\pm \mathrm{SD}$ ), white to white and axial length in the subgroup was $3.44 \pm 0.26,11.82 \pm 0.24$ and 
A
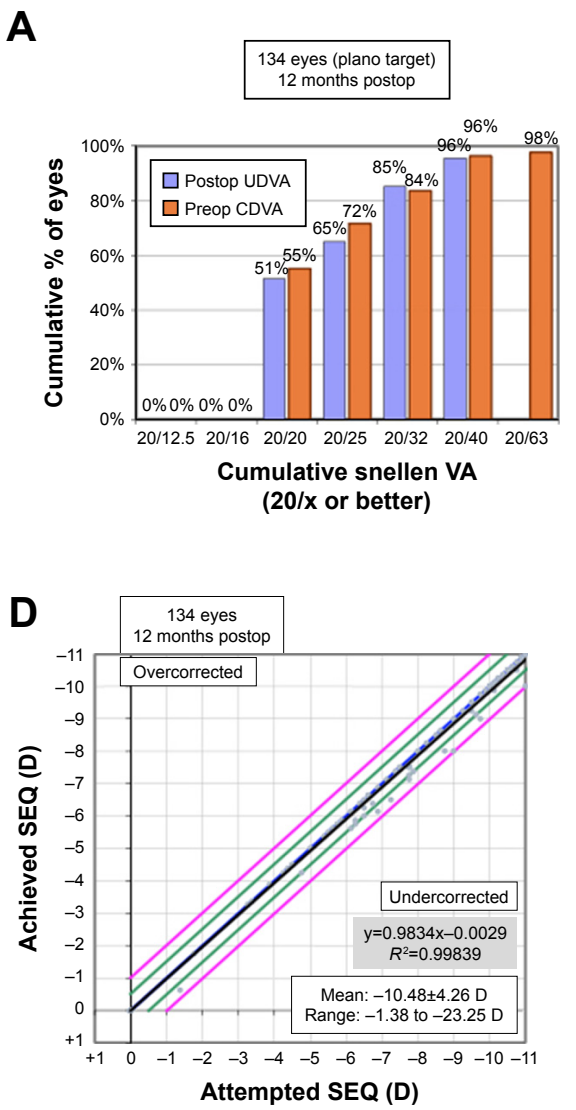

B

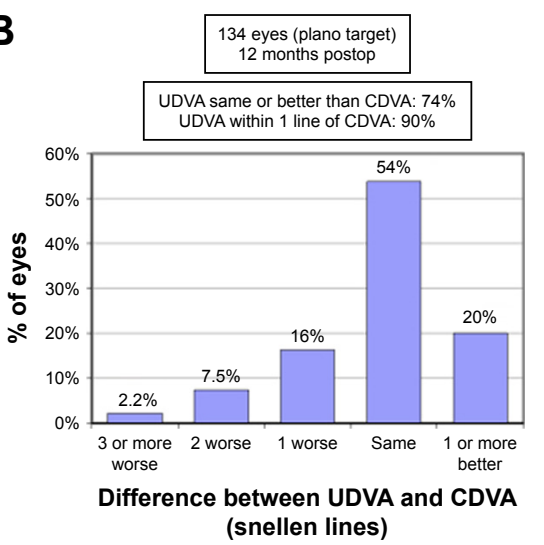

E
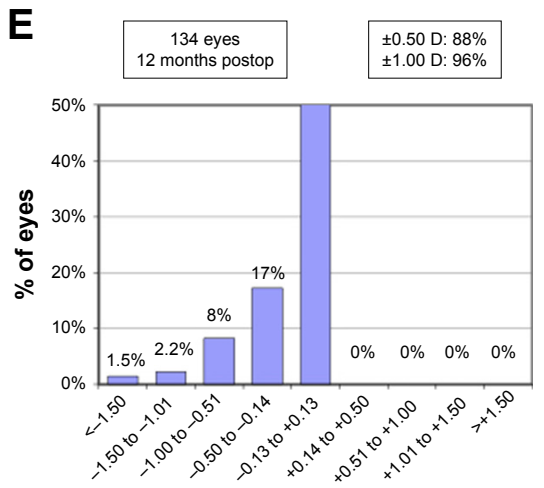

Accuracy of SEQ to

intended target (D)

G

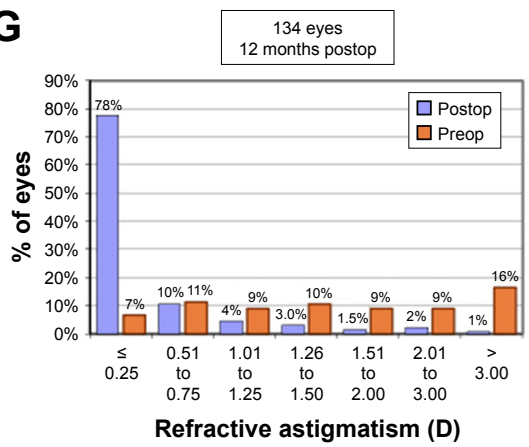

Refractive astigmatism (D)
C
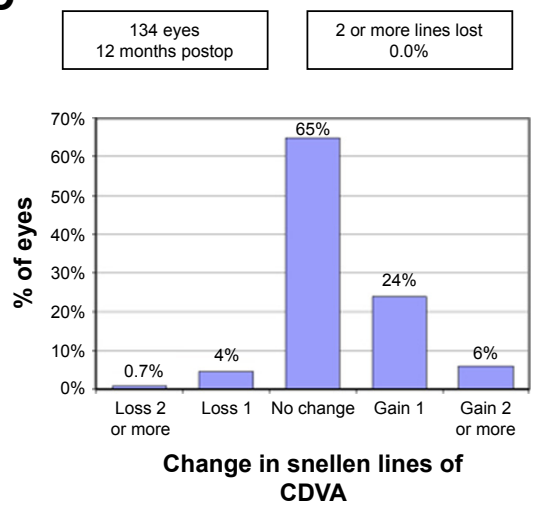

$\mathbf{F}$
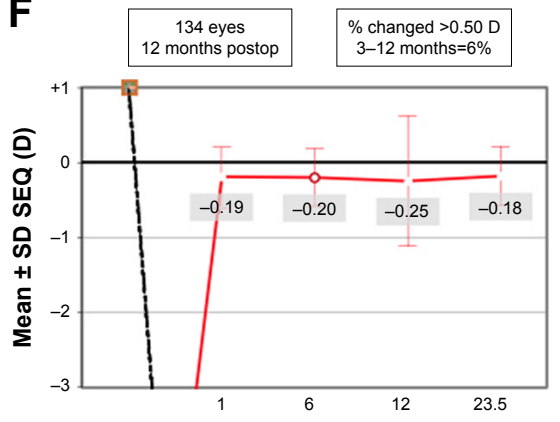

Time after surgery (months)

Figure I Refractive outcomes following IPCL implantation.

Notes: (A) UDVA. (B) UDVA vs CDVA. (C) Change in CDVA. (D) Spherical equivalent refraction attempted vs achieved. (E) Spherical equivalent refraction accuracy. (F) Spherical equivalent refraction stability. (G) Refractive astigmatism.

Abbreviations: CDVA, corrected distance visual acuity; IPCL, implantable phakic contact lens; preop, preoperative; postop, postoperative; SEQ, spherical equivalent; UDVA, uncorrected distance visual acuity.

$26.20 \pm 1.97 \mathrm{~mm}$, respectively. Four eyes underwent rotation within 14 postoperative days (mean $7.25 \pm 4.5$ days, range 2-13 days).

Five eyes underwent repositioning of the implant, subsequent to which the UDVA improved from $0.32 \pm 0.11$ $\log$ MAR to $0.12 \pm 0.11 \log$ MAR, with a reduction in absolute manifest spherical equivalent from $1.14 \pm 0.52$ to $0.29 \pm 0.20 \mathrm{D}$.
No vision threatening complications including endophthalmitis or retinal detachment were noted in the cohort.

\section{Discussion}

Phakic intraocular lens implantation has been established as an effective modality for the correction of ammetropia. This technique affords a greater range of correction in comparison to keratorefractive procedures. Additionally, superior optical 
aberration profile, no risk of regression and reversibility are other advantages. However, the invasive character of the technique increases the propensity for complications such as cataract formation, infections like endophthalmitis and endothelial cell loss.

Cataract formation and raised IOP are the most commonly documented safety concerns related to the phakic posterior chamber intraocular implant. ${ }^{9}$ Our cohort demonstrated results similar to previous studies with the ICL. The lack of a central hole in the IPCL model (V1) mandates the construction of a peripheral iridectomy, preoperative or intraoperative. Pupillary block glaucoma secondary to inadequate iridectomy can result in rise of IOP. Cataract formation is most commonly secondary to direct contact between the implant and crystalline lens or due to an altered aqueous humor circulation with subsequent lens malnutrition. Earlier studies demonstrate higher risk of cataract with older age (greater than 40 years) and higher levels of ametropia (greater than $-10.0 \mathrm{D}) .^{10-12}$

The mean age and preoperative manifest refraction was higher in the cohort, in agreement with earlier studies. As the vault sizing was adequate in all eyes (mean 369.75 $\pm 75.65 \mu \mathrm{m}$, range 259-429), the anterior subcapsular opacification was most likely secondary to metabolic changes resulting in fibrous metaplasia of the lens epithelial cells. ${ }^{13,14}$ Kawamorita et al, using computational analysis, demonstrated an increase in the velocity of aqueous humor in the central hole ICL model. ${ }^{15}$ The lack of a central optic hole in the IPCL could potentially cause a greater disturbance in the aqueous circulation, resulting in an increased incidence of cataract. The new version of the IPCL (IPCL V2), containing a 350- $\mu \mathrm{m}$ central artificial hole, has been made commercially available recently. This would obviate the need for a peripheral iridectomy and possibly bring down the incidence of cataract and pupillary block glaucoma. Studies comparing the outcomes of hole and non-hole IPCL are needed to demonstrate the same.

ECD loss was similar to data published with ICL, with a mean ECD loss ranging from $0.3 \%$ to $7.8 \%$ in previous studies. ${ }^{16-18}$ Similarly, incidence of endophthalmitis and retinal detachment was similar to ICL with a rate of $0.0167 \%$ in published literature. ${ }^{19}$

This study has certain limitations. The construct of the study was a retrospective analysis. A prospective, randomized study would be ideal for confirming our results. Second, the ECD measurements were obtained for the central cornea only. Goukon et al compared the endothelial cell loss and morphology following implantation of two different models of ICL and concluded significantly lower ECD in the superior cornea, in the non-hole cohort. ${ }^{20}$ This was attributed to the effect of the preoperative peripheral iridectomy performed in the conventional ICL group. No such comparisons were made in our cohort. Moreover, for the purpose of analysis, patients who completed a 1-year follow-up were only included in this study. Since the patients who are satisfied with their visual performance after refractive surgery tended to be lost to follow-up, our longitudinal data may have a possible source of selection bias.

\section{Conclusion}

IPCL is a safe and effective modality and may be an economically viable option for the treatment of myopia and myopic astigmatism.

\section{Author contributions}

The manuscript has been read and approved by all authors, the requirements for authorship in this document have been met, and the authors believe that the manuscript represents honest work. All authors contributed toward data analysis, drafting and critically revising the paper, gave final approval of the version to be published, and agree to be accountable for all aspects of the work.

\section{Disclosure}

The authors report no conflicts of interest in this work.

\section{References}

1. Kamiya K, Igarashi A, Shimizu K, Matsumura K, Komatsu M. Visual performance after posterior chamber phakic intraocular lens implantation and wavefront-guided laser in situ keratomileusis for low to moderate myopia. Am J Ophthalmol. 2012;153(6):1178-1186.

2. Sanders D, Vukich JA. Comparison of implantable collamer lens (ICL) and laser-assisted in situ keratomileusis (LASIK) for low myopia. Cornea. 2006;25(10):1139-1146.

3. Igarashi A, Kamiya K, Shimizu K, Komatsu M. Visual performance after implantable collamer lens implantation and wavefront-guided laser in situ keratomileusis for high myopia. Am J Ophthalmol. 2009; 148(1):164-170.

4. Pineda-Fernández A, Jaramillo J, Vargas J, Jaramillo M, Jaramillo J, Galíndez A. Phakic posterior chamber intraocular lens for high myopia. $J$ Cataract Refract Surg. 2004;30(11):2277-2283.

5. Sanders DR, Doney K, Poco M; ICL in Treatment of Myopia Study Group. United States Food and Drug Administration clinical trial of the Implantable Collamer Lens (ICL) for moderate to high myopia: threeyear follow-up. Ophthalmology. 2004;111(9):1683-1692.

6. Igarashi A, Shimizu K, Kamiya K. Eight-year follow-up of posterior chamber phakic intraocular lens implantation for moderate to high myopia. Am J Ophthalmol. 2014;157(3):532-539.

7. Kamiya K, Shimizu K, Igarashi A, Hikita F, Komatsu M. Four-year follow-up of posterior chamber phakic intraocular lens implantation for moderate to high myopia. Arch Ophthalmol. 2009;127(7):854-850.

8. Alfonso JF, Baamonde B, Fernández-Vega L, Fernandes P, GonzálezMéijome JM, Montés-Micó R. Posterior chamber collagen copolymer phakic intraocular lenses to correct myopia: five-year follow-up. J Cataract Refract Surg. 2011;37(5):873-880. 
9. Fernandes P, González-Méijome JM, Madrid-Costa D, Ferrer-Blasco T, Jorge J, Montés-Micó R. Implantable collamer posterior chamber intraocular lenses: a review of potential complications. J Refract Surg. 2011; 27(10):765-776.

10. Alfonso JF, Lisa C, Abdelhamid A, Fernandes P, Jorge J, Montés-Micó R. Three-year follow-up of subjective vault following myopic implantable collamer lens implantation. Graefes Arch Clin Exp Ophthalmol. 2010;248(12):1827-1835.

11. Gonvers M, Bornet C, Othenin-Girard P. Implantable contact lens for moderate to high myopia: relationship of vaulting to cataract formation. J Cataract Refract Surg. 2003;29(5):918-924.

12. Sanders DR. Anterior subcapsular opacities and cataracts 5 years after surgery in the visian implantable collamer lens FDA trial. $J$ Refract Surg. 2008;24(6):566-570.

13. Shiratani T, Shimizu K, Fujisawa K, Uga S, Nagano K, Murakami Y. Crystalline lens changes in porcine eyes with implanted phakic IOL (ICL) with a central hole. Graefes Arch Clin Exp Ophthalmol. 2008; 246(5):719-728.

14. Fujisawa K, Shimizu K, Uga S, et al. Changes in the crystalline lens resulting from insertion of a phakic IOL (ICL) into the porcine eye. Graefes Arch Clin Exp Ophthalmol. 2007;245(1):114-122.

15. Kawamorita T, Shimizu K, Shoji N. Effect of hole size on fluid dynamics of a posterior-chamber phakic intraocular lens with a central perforation by using computational fluid dynamics. Graefes Arch Clin Exp Ophthalmol. 2016;254(4):739-744.
16. Alfonso JF, Baamonde B, Fernández-Vega L, Fernandes P, GonzálezMéijome JM, Montés-Micó R. Posterior chamber collagen copolymer phakic intraocular lenses to correct myopia: five-year follow-up. J Cataract Refract Surg. 2011;37(5):873-880.

17. Lee J, Kim Y, Park S, et al. Long-term clinical results of posterior chamber phakic intraocular lens implantation to correct myopia. Clin Exp Ophthalmol. 2016;44(6):481-487.

18. Shimizu K, Kamiya K, Igarashi A, Kobashi H. Long-term comparison of posterior chamber phakic intraocular lens with and without a central hole (hole ICL and conventional ICL) Implantation for Moderate to High Myopia and Myopic Astigmatism: Consort-Compliant Article. Medicine. 2016;95(14): 3270.

19. Allan BD, Argeles-Sabate I, Mamalis N. Endophthalmitis rates after implantation of the intraocular collamer lens: survey of users between 1998 and 2006. J Cataract Refract Surg. 2009;35(4):766-769.

20. Goukon H, Kamiya K, Shimizu K, Igarashi A. Comparison of corneal endothelial cell density and morphology after posterior chamber phakic intraocular lens implantation with and without a central hole. $\mathrm{BrJ}$ Ophthalmol. 2017;101(11):1461-1465.

\section{Clinical Ophthalmology}

\section{Publish your work in this journal}

Clinical Ophthalmology is an international, peer-reviewed journal covering all subspecialties within ophthalmology. Key topics include: Optometry; Visual science; Pharmacology and drug therapy in eye diseases; Basic Sciences; Primary and Secondary eye care; Patient Safety and Quality of Care Improvements. This journal is indexed on

\section{Dovepress}

PubMed Central and CAS, and is the official journal of The Society of Clinical Ophthalmology (SCO). The manuscript management system is completely online and includes a very quick and fair peer-review system, which is all easy to use. Visit http://www.dovepress.com/ testimonials.php to read real quotes from published authors. 